\title{
Sex differences in tonsillitis
}

\author{
Alyaa Abouzied ${ }^{1}$ and Emad Massoud ${ }^{2}$ \\ ${ }^{1}$ Class of 2010, Faculty of Medicine, Dalhousie University \\ ${ }^{2}$ Department of Otolaryngology, Dalhousie University
}

\begin{abstract}
Tonsillopharyngitis is a common disease encountered by family physicians and otolaryngologists. Published literature since the 1970 s reveals that more females present with this condition and proceed to tonsillectomy compared to males. Female to male ratios published often exceed $2: 1$. The aim of this review is to highlight some of the theories proposed to explain this sex difference. We hope that by understanding sex-specific risk factors that make women more vulnerable to this disease, physicians could provide better care to their patients.
\end{abstract}

$\mathrm{A}_{\text {procedures performed in the Capital District Health }}^{\text {review of tonsillopharyngitis and tonsillectomy }}$ Authority of Nova Scotia, Canada is underway. Data from this study would update us on the female/male ratio of patients presenting with this condition and proceeding to surgical intervention in Nova Scotia. Based on published literature and the clinical observation of some otolaryngologists, one expects this ratio to exceed $2: 1$.

This observable sex difference has been reported in the literature in several developed countries since the 1970s. While the ratios reported may differ slightly, all papers agree on a female preponderance of chronic or recurrent tonsillitis in the teenager and young adult age groups. The aim of this review is to summarize the observations made by previous studies and discuss the reasons provided by the researchers for the sex difference in tonsillitis. A PubMed search using keywords such as tonsillitis, tonsillectomy, epidemiology, sex, sex differences, women, female and gender was done and only relevant articles that specifically discuss or report sex ratios were included. Non-English papers were excluded. No papers were excluded based on date of publication.

The review revealed that very few studies attempt to directly explore and research a hypothesis that explains the sex difference. Most papers propose reasons that have been previously cited as causes for sex differences in other diseases.

\section{Suggested reasons for sex differences}

Theoretically, sex differences in disease have always been attributed to genetic, biological, environmental or psychosocial factors. Below is a listing of factors that may be used to explain the female/male ratio in tonsillitis.

\section{Parental attitudes}

In a 1982 published study, age and sex-specific tonsillectomy rates of patients from 1970 to 1977 in the United States were compared $^{1}$. The age-specific rates in 1977 reveal a female dominance, confined to the ages 9-19 years for tonsillectomy and adenoidectomy and 9-29 years for tonsillectomy alone. At all other ages, males and females have similar rates. The female/male ratio for tonsillectomy rates for ages 9-29 was 1.9 in 1970 and 2.6 in 1977. The authors comment that while parental attitudes or sex differences in the occurrence of conditions used to justify surgery might account for the differentials, they have no data to support either explanation.

\section{Sex steroids and cultural differences}

A study of 2576 British patients from 1980-1985 undergoing tonsillectomy revealed a female preponderance in the ages 5-25 years with the highest female/male ratio of 4.08 in the band of 15-19 year olds ${ }^{2}$. The study also reported tonsillectomy rates for 272 Indian patients living in Britain and found the overall female/male ratio to be 0.87 compared to 1.58 in the British group. The authors express that the female preponderance in the Bristish population is difficult to explain and point out that cultural differences might explain the discrepancy found in the Indian population. They state that theoretically tonsillitis should be more common in males since they may have a weaker immune system and since that is not the case tonsillitis may be a manifestation of a hyperimmune state augmented by the effects of estrogen in females. The role of estrogen and testosterone in regulating both humoral and cellular immunity will be discussed in more detail below.

\section{Endocrine phenomena during puberty}

The frequency of tonsillectomy operations was estimated by a questionnaire sent to 819 individuals randomly selected 
from the Finnish Population Registry ${ }^{3}$. The paper found that the frequency of tonsillectomies was higher among female than male subjects aged between 10 and 29 years. The paper also found that in all age groups, chronic tonsillitis was the indication for surgery in more women than men. The authors conclude that the unequal sex distribution in the cause of tonsillar disease suggests that tonsillar disease is slightly different in the female than in the male patient and that endocrine phenomena occurring during puberty may explain the female preponderance among teenagers.

Data about the role of estrogen in up-regulating cellular and humoral immunity as well as the anti-inflammatory role of androgens has mostly come from the study of autoimmune diseases. In both sexes, adrenal hormones such as glucocorticoids, dehydroepiandrosterone (DHEA), and androgens, are low in patients with autoimmune diseases when compared to healthy controls. The administration of $17 \beta$-estradiol increases immune stimuli-induced secretion of cytokines such as TNF, IL-2, IL-4, IL-6, IL-10, and IFN $\gamma$ from the peripheral blood leucocytes of healthy males. Testosterone, in contrast, inhibits IL-2, IL-4 and IL-10 or tends to inhibit stimulated secretion of cytokines TNF and IFN $\gamma^{4}$. Given this information, it would seem reasonable to consider the role of sex steroids in the immunology of tonsillitis.

\section{Exposure of mothers to URTIs of children}

A group in Cape Town, South Africa performed a retrospective audit of 1991 to 1995 comparing the sex and age of patients undergoing tonsillectomy with patients admitted to hospital for acute tonsillitis and the general population. When the data for the adult tonsillectomy patients was isolated, the female/ male ratio was 3.22 (Cornfield's 95\% confidence limit: 2.82, $3.68)^{5}$. The authors' proposed explanation was that women spend more time with children suffering from pharyngitis and hence are more at risk of being infected with pathogens.

\section{Genetics}

Two retrospective questionnaires were sent to 9479 Norwegian twins, born between 1967 and 1979 and identified through the Medical Birth Registry in 1992 and 19986. The lifetime self-reported prevalence of recurrent tonsillitis was found to be $11.7 \%$ (95\% confidence interval, $11.0 \%-12.3 \%$ ), with a significant predominance of female cases. The female prevalence was $14.1 \%$ (95\% confidence interval, $13.1 \%-15.0 \%$ ) and the male prevalence was $8.8 \%$ (95\% confidence interval, 7.9\%-9.6\%) giving a female/male lifetime prevalence ratio of 1.6. The aim was to estimate the relative contribution of genetic and environmental effects in the liability of recurrent tonsillitis.
The authors assumed that there was an underlying normally distributed liability to recurrent tonsillitis but that the disease manifests only when a certain threshold is reached. Structural equation modelling was used to perform twin data analysis and the models were expanded to test for sex differences. The model that assumed no sex difference in the genetic source or genetic magnitude of variance provided the best fit for the observed data suggesting that there are no sex-specific genetic effects on the liability of recurrent tonsillitis.

The study did conclude that genetic effects, both dominant and additive, account for $62 \%$ of the variation in liability of recurrent tonsillitis. They also report that the sex difference in thresholds is statistically significant and that the threshold for manifesting the disease does not vary among different zygosity groups within a single sex. In short, the authors found no evidence to implicate genetics as a cause for the lower threshold for expressing tonsillitis in females.

\section{Severity of symptoms and social issues}

Patients with acute tonsillitis present with fever, sore throat, foul breath, dysphagia (difficulty swallowing), odynophagia (painful swallowing), and tender cervical lymph nodes. Airway obstruction may manifest as mouth breathing, snoring, nocturnal breathing pauses, or sleep apnea. Lethargy and malaise are common. Symptoms usually resolve in 3-4 days but may last up to 2 weeks despite adequate therapy. Recurrent tonsillitis is diagnosed when an individual has 7 episodes in 1 year, 5 infections in 2 consecutive years, or 3 infections each year for 3 years consecutively ${ }^{7}$. Patients with chronic tonsillitis present with chronic sore throat, halitosis, and persistent tender cervical nodes. The literature search did not reveal any study comparing the severity of these specific symptoms between the sexes.

A review paper exploring the sex differences in all respiratory tract diseases concluded that males develop RTIs more frequently than females, except for sinusitis, otitis externa, and probably tonsillitis ${ }^{4}$. The authors claim that socially defined sex roles can explain the findings. They state that the perception of disease severity may be different between males and females and that women tend to seek health care for milder diseases, such as tonsillitis and pharyngitis.

Sex differences in pain during adulthood have been researched and reviewed with data consistently showing that females are more sensitive to nociceptive stimuli ${ }^{8}$, including those that occur in internal organs ${ }^{9}$. It would be interesting to determine if women rate the severity of an episode of acute tonsillitis with the same presentation higher than men.

A 2007 published study that used surface electromyography to trace changes in oral and throat muscle activity in adults with acute and recurrent tonsillitis found that there was no 
statistically significant sex-related difference in the duration and mean muscle activity of investigated patients ${ }^{10}$. This study found that acute and recurrent tonsillitis affect muscle activity by involving additional muscles (mainly infrahyoid) in swallowing. These changes do not persist after resolution of the acute episode. However, recurrent tonsillitis affects masseter and laryngeal strap muscles, even during periods of remission, suggesting permanent pathological changes. Since chronic tonsillitis is more prevalent in women, one can conclude that the complications of tonsillitis such as painful swallowing, even during remission, are probably more common among women.

\section{Sexually transmitted infections}

The practice of fellatio by women has increased ${ }^{11}$. For some, fellatio can be a substitute to vaginal intercourse to preserve "virginity" 12 . Oral sex can expose the oropharynx to bacteria that are not part of the normal flora and may have an effect on the manifestation of tonsillitis.

A 2003 review of oral gonococcal pharyngitis found that the most important risk factor for developing oral gonococcal infection was the practice of fellatio by heterosexual women and homosexual men ${ }^{13}$. Although heterosexual men with positive culture for gonococci from the oropharynx were also reported in the literature, the link with the practice of cunnilingus seems less clear. Oral gonococcal infection can occur in the absence of genital infection but the symptoms may be subtle or absent and hence can be easily missed. The review concluded that physicians must include primary gonococcal pharyngitis in their differential diagnosis of acute exudative tonsillitis in young sexually active women.

Chlamydia trachomatis is the most common bacterial sexually transmitted pathogen in developed countries. A 1995 study of the value of screening for oropharyngeal Chlamydia trachomatis found that the prevalence of this bacterium was very low, did not pose a serious health risk and hence was not worthwhile ${ }^{14}$. Another 1994 study found that the rate of genital mycoplasma isolation was significantly higher in children with recurrent adenotonsillitis $(34.5 \%)$ than in those with obstructive symptoms $(3.7 \%)$. They argue that further studies are needed to evaluate the possible pathogenic role of these microorganisms in adenotonsillar infection ${ }^{15}$.

Little research has been conducted concerning other organisms that might colonize the oropharynx of women practising oral sex. A better understanding of the link between fellatio and recurrent tonsillitis can help identify populations that are at high risk for this disease. It is important to realize that data from different cultures can be very different depending on the prevalence of this sexual practice.

\section{Benefit from tonsillectomies}

A 2005 paper assessed the benefit of tonsillectomy in adult patients with chronic tonsillitis using a questionnaire ${ }^{16}$. The results show that younger patients reported more improvement in quality of life after surgery compared to older patients. Patient gender did not influence the rating of benefit suggesting that while women might be more likely to proceed with a tonsillectomy due to more chronic tonsillar disease, they do not regard outcomes post surgery any different than men.

\section{Conclusion}

Tonsillitis is one of the most common complaints seen by family doctors and otolaryngologists. The sex difference in chronic and recurrent tonsillitis and accordingly tonsillectomy surgeries has been an unexplained observation for the past 30 years. Many reasons have been proposed over the years but the list is by no means comprehensive. Guidelines for establishing a diagnosis of tonsillitis may have been introduced later than most of the published data and criteria for tonsillectomy procedures have been refined over time. It would be interesting to see if having uniform practice guidelines among different ENT specialists would eliminate this sex difference. An understanding of sex-specific risk factors that make women more vulnerable would possibly help decrease the incidence and/or improve the management of this disease as well as reduce the need for tonsillectomy.

\section{REFERENCES}

1. Freeman JL, Jekel JF, Freeman DH, Jr. Changes in age and sex specific tonsillectomy rates: United states, 1970-1977. Am J Public Health. 1982;72:488-491.

2. Moloney JR. Age, sex, ethnic origin and tonsillectomy. The journal of laryngology and otology. 1988;102:649.

3. Mattila PS, Tahkokallio O, Tarkkanen J, Pitkaniemi J, Karvonen M, Tuomilehto J. Causes of tonsillar disease and frequency of tonsillectomy operations. Arch Otolaryngol Head Neck Surg. 2001;127:37-44.

4. Falagas ME, Mourtzoukou EG, Vardakas KZ. Sex differences in the incidence and severity of respiratory tract infections. Respiratory Medicine. 2007; 101:1845-1863.

5. Thorp MA, Isaacs S, Sellars SL. Tonsillectomy and tonsillitis in cape town--age and sex of patients. S Afr J Surg. 2000;38:62-64.

6. Kvestad E, Kvaerner KJ, Roysamb E, Tambs K, Harris JR, Magnus P. Heritability of recurrent tonsillitis. Arch Otolaryngol Head Neck Surg. 2005; 131:383-387.

7. Udayan K shah. Tonsillitis and Peritonsillar Abscess. E-Medicine Website. http://www.emedicine.com/Ent/topic314.htm. Updated January 16, 2008. Accessed January 16, 2008.

8. Fillingim RB, Ness TJ. Sex-related hormonal influences on pain and analgesic responses. Neurosci Biobehav Rev. 2000;24:485-501.

9. Giamberardino MA. 2000. sex-related and hormonal modulation of visceral pain, pp. 135-163. in: Sex, Gender and Pain. 2000:135-163.

10. Vaiman M. The influence of tonsillitis on oral and throat muscles in adults. Otolaryngology - Head and Neck Surgery. 2007;136:832-837.

11. Evans B, McCormack S, Kell P, Parry J, Bond R, MacRae K. Trends in female sexual behaviour and sexually transmitted diseases in london, 1982-1992. Sex Transm Infect. 1995;71:286-290.

12. Sanders SA, Reinisch JM. Would you say you "had sex" if... ? JAMA. 1999;281:275-277.

13. Balmelli C, Gunthard HF. Gonococcal tonsillar infection--a case report and literature review. Infection. 2003;31:362-365. 
14. Jebakumar SP, Storey C, Lusher M, Nelson J, Goorney B, Haye KR. Value of screening for oro-pharyngeal chlamydia trachomatis infection. J Clin Pathol. 1995;48:658-661.

15. Huminer D, Pitlik S, Levy R, Samra Z. Mycoplasma and chlamydia in adenoids and tonsils of children undergoing adenoidectomy or tonsillectomy. Ann Otol Rhinol Laryngol. 1994;103:135-138.

16. Baumann I, Kucheida H, Blumenstock G, Zalaman IM, Maassen MM, Plinkert PK. Benefit from tonsillectomy in adult patients with chronic tonsillitis. Eur Arch Otorhinolaryngol. 2006;263:556-559.

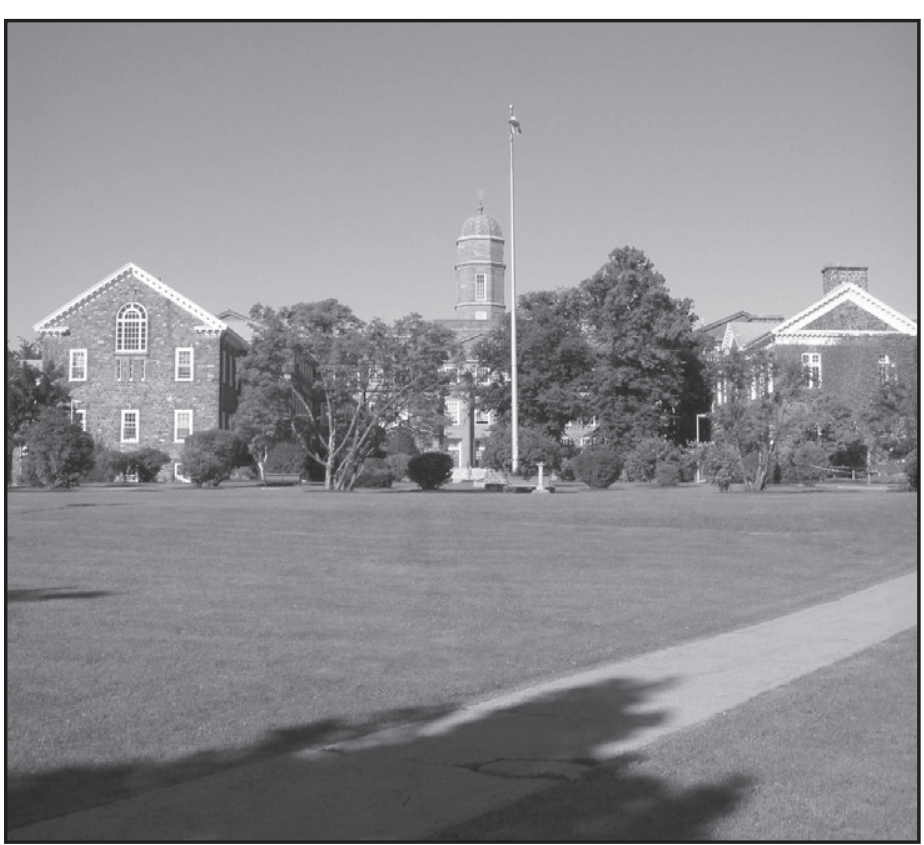

\section{Cape Breton Island Great People, Great Place, Great Life}

The Cape Breton District Health Authority requires:

\section{General Practitioners and Specialists}

Through regional, community and rural hospitals, Continuing Care, Mental Health Services, Addiction Services, Public Health Services and the Cape Breton Cancer Centre, the District serves more than 130,000 people. Cape Breton's unique beauty, diverse culture and year-round recreational opportunities make the Island a vibrant place to practice and play.

All candidates must be certified by the Royal College of Physicians and Surgeons and be eligible for licensure in the province of Nova Scotia.

Inquiries and applications to:

Dr. M.A. Naqvi, Medical Director

Cape Breton District Health Authority

1482 George Street

Sydney, Nova Scotia B1P 1 P3

Fax: (902) 567-7921

E-mail: naqvim@ cbdha.nshealth.ca

Website: www.cbdha.nshealth.ca

* Relaxed Lifestyle

* Nurturing Communities

* Affordable Homes

* www.cbisland.com

* www.capebretonisland.com

\section{You've already decided to make your living caring for others.}

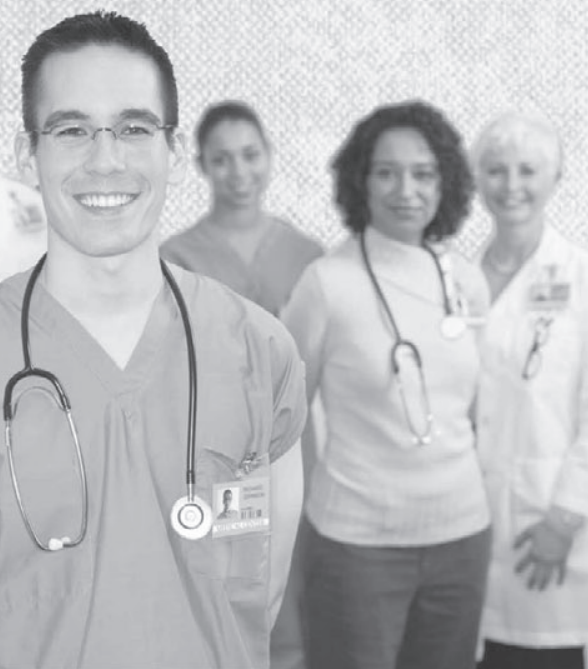

Now you just have to choose where.

\section{Come to Capital Health, Nova Scotia}

Where patient care comes first - and is second to none.

Where you'll build a rewarding career with world-class

health care colleagues.

Where academic and research opportunities abound at nationally recognized Dalhousie University.

Where the Atlantic Ocean is close enough for you to dip your toe... while on your lunch break.

Where you'll wonder what took you so long to get here.

To learn about the choices and opportunities at Capital Health, please contact:

Capital Health Tel: (902) 473-4313 Email: david.osmond@cdha.nshealth.ca or visit www.cdha.nshealth.ca 